\title{
Hemodynamic phenotypes and its association with blood pressure changes at continuous positive airway pressure therapy in obstructive sleep apnoea hypertensive patients
}

\author{
Raissa Khursa', Margarita Voitikova², Adrian Stefański ${ }^{3}$, Jacek Wolf ${ }^{3}$, Krzysztof Narkiewicz ${ }^{3}$ \\ 'Belarusian State Medical University, Minsk, Belarus \\ ${ }^{2}$ Institute of Physics, National Academy of Science, Minsk, Belarus \\ ${ }^{3}$ Medical University of Gdańsk, Faculty of Medicine, Department of Hypertension and Diabetology, Gdańsk, Poland
}

\begin{abstract}
Background. The goal of our study was to define the hemodynamic phenotypes in hypertensive patients with newly diagnosed obstructive sleep apnoea (OSA) using the individual modelling of hemodynamics derived from ambulatory blood pressure monitoring (ABPM), a method proposed by our group previously, and to assess its validity in blood pressure alterations secondary to continuous positive airway pressure (CPAP).

Material and methods. Thirty-three hypertensive patients with moderate-to-severe OSA were investigated. All patients underwent $\mathrm{ABPM}$ on two occasions: at baseline and after one week CPAP. The sets of BP indexes at first ABPM were used for individual modelling to define the hemodynamic phenotype (class) based on regression analyses; specifically, the phenotypes were defined for daytime, nighttime and for 24 hours. The CPAP therapy efficacy was predefined as improvement in BP nighttime decrease for additional $5 \%$ as compared to baseline ABPM. With reference to this criterion, patients were further classified as responders (who achieved this target), and non-responders. Results. Only $21.2 \%$ of hypertensive patients with OSA had optimal hemodynamic phenotype (class H2), despite comprehensive antihypertensive therapy; most of the other patients were classified either as harmonic type (class $\mathrm{H} 3$; $39.4 \%$ ), or of diastolic dysfunctional type (class D3; 15.2\%). In the daytime period $18.2 \%$ of patients with OSA were classified as D1-class, which is associated with high risk of acute hypotensive episodes. Responders were more frequently characterized by phenotype $\mathrm{H} 3$ (daytime) vs. nonresponders: $53.3 \%$ and $5.6 \%$ respectively; $\mathrm{P}<0.05$. At nighttime non-responders were more likely to transform the different baseline classes into $\mathrm{H} 3$ and $\mathrm{D} 2 ; \mathrm{P}<0.05$ vs. responders. Conclusion. Our study suggest utility of newly developed modelling based on hemodynamic BP indexes in the prediction of BP alterations secondary to CPAP in OSA hypertensive patients.

Key words: obstructive sleep apnoea, blood pressure, regression modelling, hemodynamic phenotypes, CPAP-therapy. Arterial Hypertens. 2018, vol. 22, no. 3, pages: 113-119 DOI: $10.5603 /$ AH.a2018.0011
\end{abstract}

\section{Introduction}

Obstructive sleep apnoea (OSA) is a condition with recurring episodes of full (apnoea) or partial (hypo- pnea) upper airway obstruction during sleep. It leads to breathlessness or to significant decrease in breathing amplitude, resulting in a desaturation of blood and tissue hypoxia, as well as to sleep defragmenta-

Address for correspondence: Raissa Khursa

Belarusian State Medical University,

Minsk, Belarus

e-mail: rvkhursa@tut.by 
tion. Currently the method of choice for OSA treatment consists of continuous positive airway pressure (CPAP) [1].

There are many studies confirming the relationship of OSA with hypertension, coronary heart disease, arrhythmias, chronic heart failure and stroke, overall and cardiovascular mortality [2-7]. Therefore, cardiologic aspects of OSA are very relevant. OSA is frequently associated with the inverse of 24-hour blood pressure (BP) profile as well as nocturnal and excessive morning BP rise that could be one of the mechanisms responsible for increased cardiovascular risk in this group. Therefore, an improvement in nocturnal BP dipping may be an important clinical goal in patients with OSA.

Contemporary statistical methods (Data Mining) allow for expanded evaluation of ABPM-derived data. In particular, a BP level is the important hemodynamic indicator, integrating left ventricle's and vessels' work. It is well established that blood flow is ensured by the concerted efforts of the heart and the vessels, but their participation in the process of blood moving varies in different people, namely in different of the heart volume ratio per minute and of the total peripheral resistance. We have used the original functional diagnostics of hemodynamics on individual series of the patient's BP indexes received during desired observation period on linear regression of systolic (SBP) and diastolic (DBP) blood pressure on pulse pressure $W$.

Therefore, the goal of our study was to evaluate hemodynamic phenotypes of OSA-hypertensive patients with our novel technique. Additionally, we sought to investigate the potential clinical application of IMH-BP-based data in OSA treated patients with positive airway pressure.

\section{Material and methods}

A total of 33 patients ( 5 females; mean age 60.4 \pm 1.5 years; hypertension duration $17.4 \pm 1.7 \mathrm{y}$ [mean $\pm S D]$ ) with moderate-to-severe OSA were included in the study. The patients were overweight or obese - median of the body mass index (BMI) was $33.0[30.3-39.1] \mathrm{kg} / \mathrm{m}^{2}$ (median [IQR]) and were free from concomitant cardiovascular diseases. All patients received antihypertensive treatment (the number of drugs was $1-7$, mean $-4.1 \pm 0.6$ ). The diagnosis of OSA was based on sleep-time polygraphy recordings $\left(\right.$ Embletta $\left.^{\mathrm{TM}} \mathrm{X} 30\right)$ with inclusion criterion of respiratory event index (REI) of 15 or greater, within a month prior to ABPM studies (REI
= 32.4 [17.9-43.5] episodes/hour; median [IQR]). All patients underwent ABPM on two occasions: at baseline and within the first week of CPAP therapy. The sets of BP indexes at first ABPM in every patient were used for linear regression of BP parameters to define the hemodynamic phenotypes (classes). The phenotypes were defined for daytime, nighttime and for 24 hours. The CPAP therapy efficacy was predefined as improvement in BP nighttime decrease for additional $5 \%$ as compared to baseline ABPM. With reference to this criterion, patients were further classified as (1) responders and (2) non-responders.

The equation for the regression analyses is as follows: $S B P=Q+a^{*} W$ and $D B P=Q+(1-a)^{*} W$; where $\mathrm{Q}$ is an intercept; $\mathrm{a}$ - angular coefficient; SBP — systolic BP; DBP - diastolic BP; W - pulse pressure (W = SBP-DBP).

In the present study we used the previously proposed classification and interpretation of hemodynamic types [8]. In short, according with the a-value patients may be classified in the following groups: (1) harmonious $(0<a<1, \mathrm{H}$-type, norm), (2) diastolic dysfunctional ( $a>1$, D-type, associated with a hypertrophied role of left ventricle in the circulation, or (3) systolic dysfunctional type ( $a<0$, S-type, a hypertrophied role of "peripheral heart», peripheral resistance increased) [8].

Such statistical modelling of the individual hemodynamic status on BP parameters received its further development by application Data Mining algorithm SVM (Support Vector Machine) to the obtained regression coefficients. The set of individual linear regression coefficients $\{Q, a\}$ for patient's BP indexes derived from 24-hour ambulatory blood pressure monitoring (ABPM) may be subsequently mapped on nomogram (Fig. 1), diagnosing up to 10 hemodynamic classes in patients with normal $\mathrm{BP}$, with hypertension $(\mathrm{AH})$ and with hypotension $[9,10]$.

From the clinical perspective, the general interpretation of the hemodynamic classes is as follows: D1 ('hypotensive diastolic dysfunctional'), D2 ('normotensive diastolic dysfunctional'), D3 ('hypertensive diastolic dysfunctional'), H2 ('normotensive harmonic'), H3 ('hypertensive harmonic') and $\mathrm{H} 0$ (or qH "quasi-hypertension" in normotensive patients, which is the borderline class between $\mathrm{H} 2$ and $\mathrm{H} 3$, and which may be diagnosed only at daytime) - see Figure 1 .

According to the individual modelling of hemodynamics on BP parameters (IMH-BP) in patients with OSA, hemodynamic phenotypes have not been defined before. 


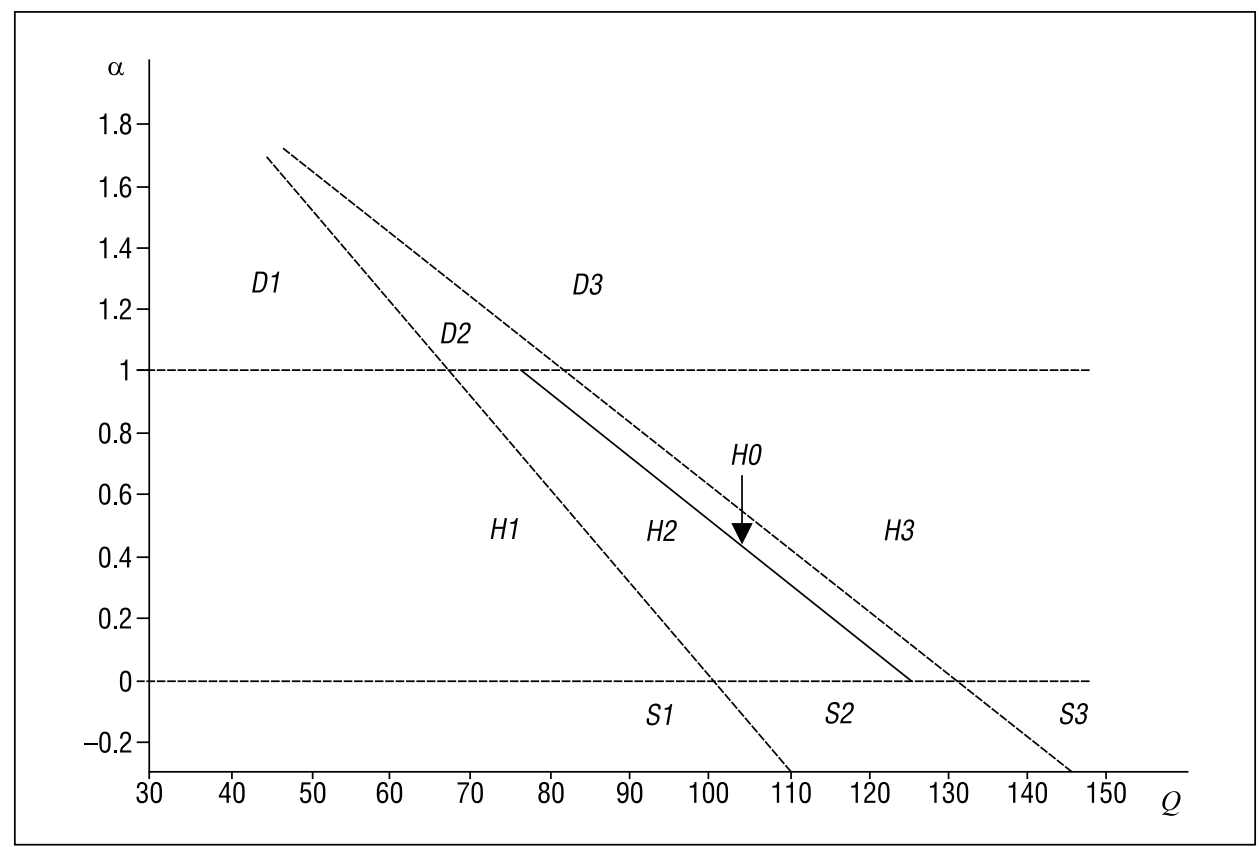

Figure 1. Nomogram (the map $\{0, a\})$ for diagnosing of hemodynamic classes (phenotypes) at the ABPM (for daytime period): D1, H1, S1 hypotensive patients; $\mathrm{D} 2, \mathrm{H} 2, \mathrm{HO}(\mathrm{qH}), \mathrm{S} 2$ - normotensive patients; $\mathrm{D} 3, \mathrm{H} 3, \mathrm{~S} 3$ - hypertensive patients

The analysis of individual sets of BP indexes from baseline ABPM included IMH-BP in every patient: the linear regression of BP parameters and the classifications of their hemodynamic class based on regression coefficients for periods separately: daytime, nighttime and 24 hours.

The CPAP therapy efficacy was evaluated on recorded logs related to flow parameters, operating time and residual AHI. Additionally, the magnitude of night dipping changes was also evaluated with repeated ABPM. The diurnal profile improvement (increase of at least 5\% dipping at night vs. baseline) allowed for grouping the patients to (1) responders subgroup and (2) non-responders. Differences between the ABPM parameters in responders/non-responders subgroups were analysed retrospectively in order to identify the relation between initial hemodynamic phenotype and hemodynamic response to CPAP therapy.

Statistical analysis was performed with Statistica 10 , Statsoft. Both, parametric and nonparametric calculations were employed where appropriate. P-value of less than 0.05 was considered valid for all tests.

\section{Results}

At baseline, none of the patients was characterized by normal diurnal BP profile (10-20\% of BP dipping at nighttime). The BP profile was $0.1 \%$
Table I. ABPM data for baseline characteristic

\begin{tabular}{|l|c|c|}
\hline \multirow{2}{*}{ Period of ABPM } & \multicolumn{2}{|c|}{ Parameters } \\
\cline { 2 - 3 } & SBP, $\mathrm{mmHg}$ & DBP, $\mathrm{mmHg}$ \\
\hline Daytime & $136.8 \pm 2.8$ & $73.3(80.7-85.8)$ \\
\hline Nighttime & $139.0 \pm 3.0$ & $71.2(77.2-85.9)$ \\
\hline 24-hour & $137.6 \pm 2.7$ & $72.1(80.4-85.4)$ \\
\hline
\end{tabular}

Data presented as mean $\pm S D$, and Me (IQR)

$(-4.7 \%$ to $+3.4 \%)$ for SBP, and $1.5 \%(-1.9 \%$ to $+6.8 \%$ ) for DBP [Me (25Q-75Q)]. Blood pressure parameters at the baseline ABPM are presented in Table I.

The distribution of hemodynamic classes according to IMH-BP is presented in Figure 2.

Only $18.2 \%$ of patients had the optimal hemodynamic class $\mathrm{H} 2$ over the daytime, and $21.2 \%$ - over the 24-hour period, despite receiving multidrug antihypertensive treatment. Most patients were grouped as H3-class, which was most evidently, when nighttime phenotypes and 24-hour ones were assessed (Fig. 2). This fact confirms the insufficient BP control in these patients, especially at the night.

SBP and DBP at different phenotypes for every timeframe and $\mathrm{P}$-value between them are presented in Table II.

Class H3 was characterized by highest $Q$-pressure, especially at the nighttime; the classes of D-type by lowered $Q$-pressure, especially class D1. Class 
D1, which is characterized by very low pressure $Q$ (Table III) was diagnosed in $18.2 \%$ of patients in the

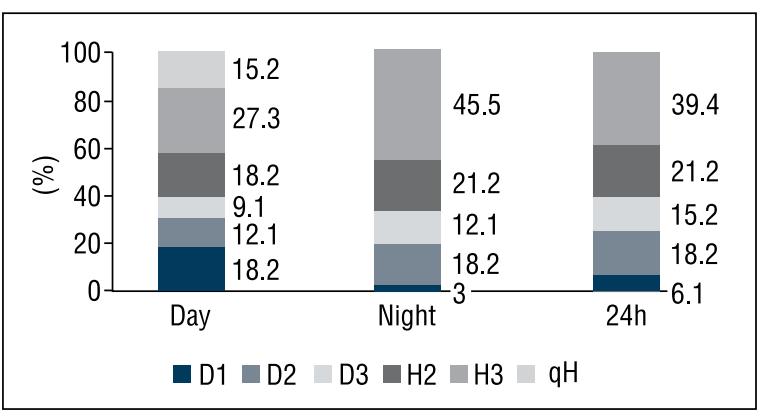

Figure 2. Frequency of the hemodynamic classes for observation periods (daytime, nighttime, 24-hour), \% daytime period. This situation is dangerous, because excessive decrease of BP during day can lead to its significant increase at the night. This class has a high risk of acute hypotensive episodes [10,11].

CPAP therapy was capable of improving diurnal profile in 15 patients, whereas 18 patients (nonresponders) had their abnormal diurnal BP profile intact.

Selected characteristic of responders and non-responders are presented in Table IV.

There were no significant differences in $\mathrm{BP}$ parameters in responders vs. non-responders, as well as between BP-values at ABPM 1 and at ABPM 2.

The patients of both group had the inverse diurnal index (non-dipping), which was partially improved after CPAP commencement (Fig. 3.)

Table II. Blood pressure in patients with OSA at different classes (hemodynamic phenotypes), mmHg, Me (250-750)

\begin{tabular}{|l|c|c|c|c|c|c|}
\hline $\begin{array}{l}\text { Class } \\
\text { (day) }\end{array}$ & SBP, day & DBP, day & SBP, 24h & DBP, 24h & SBP, night & DBP, night \\
\hline H3 & $\begin{array}{c}140.4 \\
(138.7-164.9)\end{array}$ & $\begin{array}{c}91.22 \\
(84.2-93.5)\end{array}$ & $\begin{array}{c}147.8 \\
(140.3-168.4)\end{array}$ & $\begin{array}{c}92.5 \\
(83.8-95.1)\end{array}$ & $\begin{array}{c}149.3 \\
(140.7-164.5)\end{array}$ & $\begin{array}{c}92.5 \\
(78.3-97.6)\end{array}$ \\
\hline D1 & 131.8 & 68.13 & 132.8 & 69.4 & 139.0 & 73.6 \\
& $(114.5-136.0)$ & $(65.7-73.3)$ & $(121.1-141.5)$ & $(68.3-73.8)$ & $(128.1-145.9)$ & $(68.6-76.5)$ \\
\hline qH & 128.6 & 81.5 & 129.2 & 82.0 & 129.9 & 83.0 \\
& $(126.9-128.9)$ & $(80.1-81.6)$ & $(126.6-135.5)$ & $(80.9-82.0)$ & $(126.2-131.4)$ & $(75.0-83.1)$ \\
\hline D3 & 144.6 & 91.1 & 146.5 & 90.4 & 150.1 & 89.1 \\
& $(135.0-149.8)$ & $(85.6-91.9)$ & $(116.1-123.2)$ & $(81.4-91.4)$ & $(127.4-150.9)$ & $(75.8-90.2)$ \\
\hline H2 & 120.5 & 72.0 & 118.8 & 71.0 & 115.9 & 68.5 \\
& $(120.1-123.2)$ & $(71.2-74.6)$ & $(116.1-123.2)$ & $(69.9-72.3)$ & $(112.8-123.1)$ & $(65.3-71.8)$ \\
\hline D2 & 142.7 & 78.1 & 140.4 & 77.2 & 140.4 & 75.6 \\
& $(138.1-150.7)$ & $(74.7-83.5)$ & $(126.6-144.8)$ & $(72.8-83.2)$ & $(131.7-154.2)$ & $(69.0-82.8)$ \\
\hline p-value & 0.0029 & 0.0001 & 0.0033 & 0.0002 & 0.0089 & 0.0012 \\
\hline
\end{tabular}

Table III. Regression coefficients $(a, 0)$ in patients with OSA at different hemodynamic classes, Me (250-750)

\begin{tabular}{|l|c|c|c|c|c|c|}
\hline $\begin{array}{l}\text { Class } \\
\text { day }\end{array}$ & a day & a night & $\begin{array}{l}\text { a } 24 h \\
\mathbf{O} \text { day, } \mathbf{m m H g}\end{array}$ & $\mathbf{Q}$ night, $\mathbf{m m H g}$ & $\mathbf{Q} 24 \mathrm{~h}, \mathbf{m m H g}$ \\
\hline H3 & $\begin{array}{c}0.81 \\
(0.73-0.96)\end{array}$ & $\begin{array}{c}0.6 \\
(0.29-0.86)\end{array}$ & $\begin{array}{c}0.75 \\
(0.61-0.87)\end{array}$ & $\begin{array}{c}103.6 \\
(95.5-122.5)\end{array}$ & $\begin{array}{c}126.1 \\
(98.7-129.1)\end{array}$ & $\begin{array}{c}110.6 \\
(98.4-125.3)\end{array}$ \\
\hline D1 & 1.26 & $\begin{array}{c}1.12 \\
(1.16-1.44)\end{array}$ & $\begin{array}{c}1.26 \\
(1.1-1.5)\end{array}$ & $\begin{array}{c}53.3 \\
(40.0-58.9)\end{array}$ & $\begin{array}{c}63.9 \\
(30.4-79.3)\end{array}$ & $\begin{array}{c}53.9 \\
(32.3-60.9)\end{array}$ \\
\hline qH & 0.82 & 0.71 & 0.83 & 87.8 & 93.4 & 89.2 \\
& $(0.61-0.93)$ & $(0.66-0.72)$ & $(0.52-0.9)$ & $(84.1-100.1)$ & $(90.4-98.5)$ & $(83.6-104.4)$ \\
\hline D3 & 1.1 & 1.04 & 1.11 & 81.2 & 86.5 & 83.3 \\
& $(1.08-1.17)$ & $(0.49-1.78)$ & $(0.8-1.19)$ & $(81.0-86.2)$ & $(42.5-101.9)$ & $(80.9-90.9)$ \\
\hline H2 & 0.71 & 0.70 & 0.71 & 84.6 & 81.4 & 82.5 \\
& $(0.59-0.9)$ & $(0.64-1.06)$ & $(0.61-0.95)$ & $(77.0-93.4)$ & $(62.0-86.6)$ & $(72.6-91.0)$ \\
\hline D2 & 1.22 & 1.07 & 1.16 & 66.3 & 70.7 & 68.1 \\
& $(1.12-1.26)$ & $(0.82-1.1)$ & $(1.06-1.16)$ & $(63.7-68.32)$ & $(61.0-91.4)$ & $(61.8-77.9)$ \\
\hline p- value & 0.0003 & 0.149 & 0.003 & 0.0001 & 0.0098 & 0.0002 \\
\hline
\end{tabular}


Table IV. Selected characteristics and IMH-BP parameters in responders and non-responders groups

\begin{tabular}{|l|c|c|c|c|c|c|}
\hline \multirow{2}{*}{ Characteristics } & \multicolumn{3}{|c|}{ Responders } & \multicolumn{3}{c|}{ Non-responders } \\
\cline { 2 - 7 } & Me & 250 & 750 & Me & 250 & 750 \\
\hline Age (years) & 59.0 & 52.0 & 63.0 & 62.0 & 56.0 & 69.0 \\
\hline Duration of hypertension (years) & 19.0 & 9.0 & 30.0 & 17.0 & 9.5 & 19.5 \\
\hline Antihypertensive drugs (number) & 5.0 & 3.0 & 6.0 & 3.5 & 2.0 & 5.0 \\
\hline 0, day [mmHg] & 95.5 & 81.0 & 105.0 & $78.1^{*}$ & 61.5 & 86.2 \\
\hline 0, night [mmHg] & 98.7 & 86.5 & 128.9 & $75.2^{*}$ & 58.7 & 98.5 \\
\hline 0, 24h [mmHg] & 98.3 & 79.3 & 112.3 & $77.5^{*}$ & 61.0 & 89.2 \\
\hline a, day & 0.82 & 0.59 & 0.98 & 1.06 & 0.83 & 1.19 \\
\hline a, night & 0.67 & 0.38 & 0.96 & 0.91 & 0.66 & 1.29 \\
\hline a, 24h & 0.79 & 0.61 & 1.11 & 0.96 & 0.80 & 1.17 \\
\hline
\end{tabular}

${ }^{*} p<0.05$ vs. responders

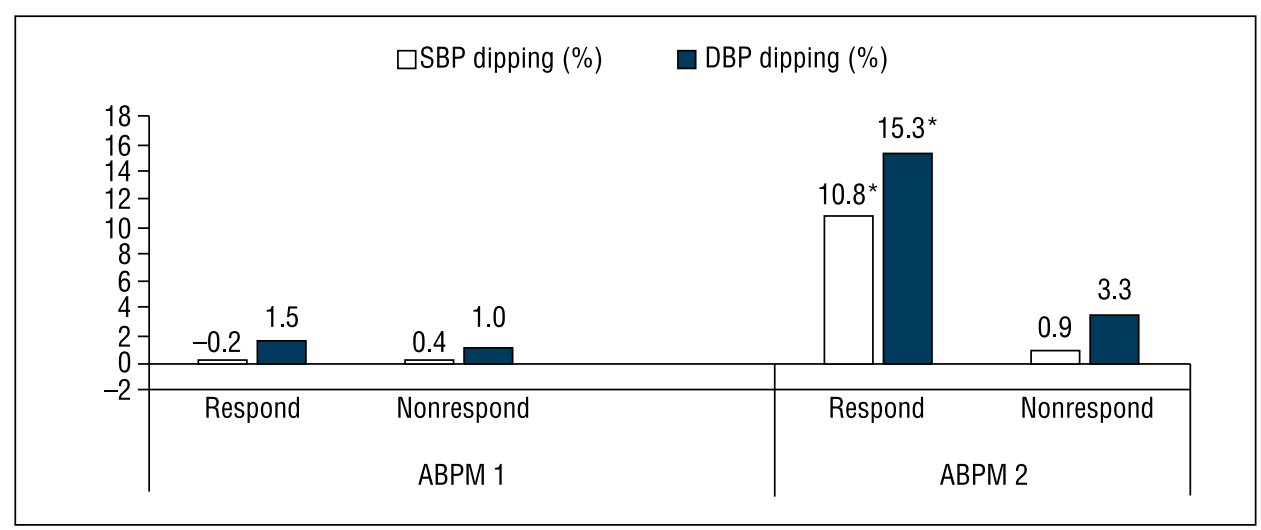

Figure 3. Diurnal index in responders and non-responders at initial ABPM and after CPAP therapy $\left({ }^{*} \mathrm{p}<0.05\right.$ vs. responders)

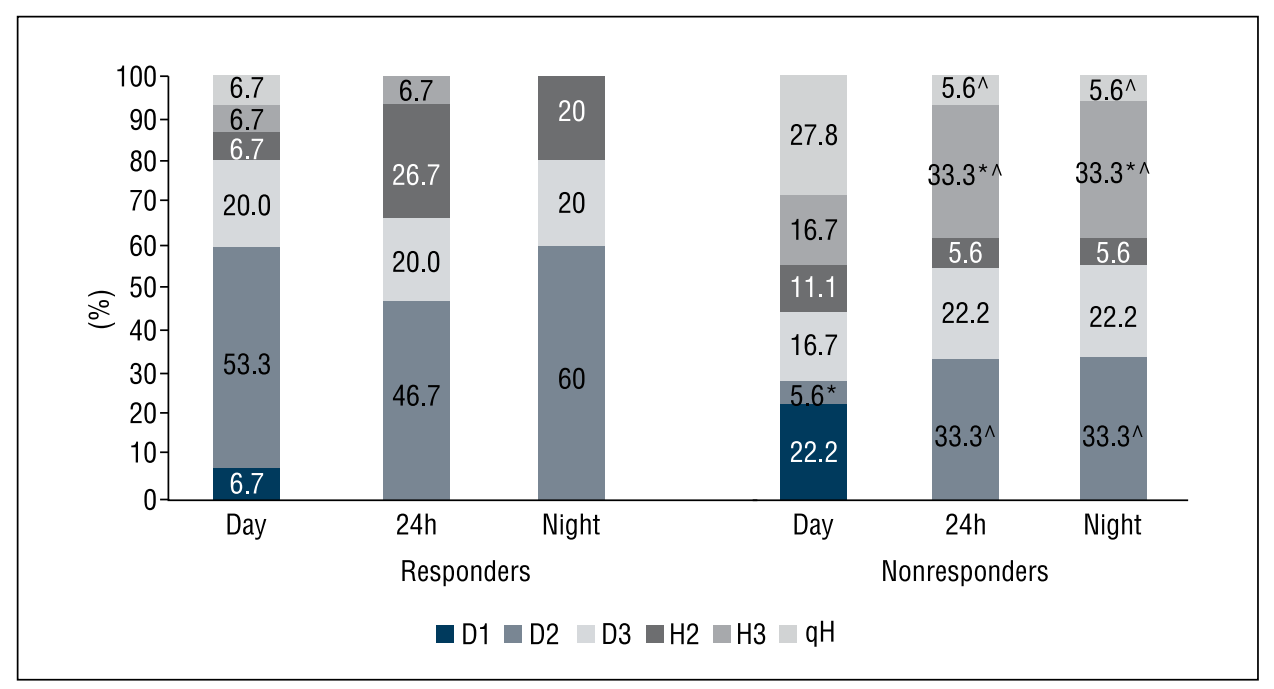

Figure 4. Hemodynamic classes during observation periods of ABPM 1 (frequency, \%); ${ }^{*} p<0.05$ vs. responders group; $\wedge p<0.05$ vs. daytime period of their group

Based on the daytime IMH-BP responders were characterized by class $\mathrm{H} 3$ phenotype which contrasted with the non-responders group: $53.3 \%$ and $5.6 \%$ respectively $\left(\chi_{2}=7.2, \mathrm{p}=0.007\right)$ (Fig. 4). 


\section{Discussion}

Our study showed that ABPM data processing with IMH-BP technique allowed for (1) the unique grouping of hypertensive OSA patients as well as (2) the identification of patients whose BP profile would improve in response to CPAP.

Individual modelling of hemodynamics on $\mathrm{BP}$ (IMH-BP) opens new circulatory characteristics, which reflect the patient's hemodynamic phenotype: type of cardiovascular interaction during blood advancement and hemodynamic class (phenotype).

Only $21.2 \%$ of hypertensive patients with OSA, receiving comprehensive antihypertensive therapy, belonged to a class of optimal hemodynamics (H2), most of the other patients kept hypertensive hemodynamics either of harmonic type (class $\mathrm{H} 3$ ) $39.4 \%$, or of diastolic dysfunctional type (class D3) $-15.2 \%$. In the daytime period $18.2 \%$ of patients with OSA have hemodynamic class D1, which is related with increased risk of acute hypotensive episodes; therefore, these patients' treatment regimen requires prompt revision.

The hemodynamic phenotype $\mathrm{H} 3$ during daytime period was more frequent in responders, than in non-responders: $53.3 \%$ and $5.6 \%$ respectively; non-responders demonstrated phenomenon of phenotypes transformation at nighttime, especially into classes $\mathrm{H} 3$ and $\mathrm{D} 2$ from other classes, $\mathrm{p}<0.05$ vs. responders.

Individual modelling of hemodynamics on $\mathrm{BP}$ at ABPM allowed us to make a selection of the patients with OSA for CPAP therapy. Namely, phenotype H3 in daytime period of ABPM is the most prospective to a positive hemodynamic response in the form of diurnal index increasing; phenotype D1 - in the form of transformation into better hemodynamic classes at night.

The patients, whose daytime models (except class H3) are transformed into class $\mathrm{H} 3$ in nighttime, would more likely present abnormal BP-diurnal pattern while on CPAP.

The non-responders demonstrated high variability of classes between observation periods - from day to night and to 24 hour: at night the number of persons with class $\mathrm{H} 3$ (harmonious hypertensive hemodynamics) increased significantly from 5.6\% to $33.3 \%\left(\chi_{2 \mathrm{MN}}=7.69 ; \mathrm{p}=0.005\right)$, what indicates negative hemodynamic tendency. However, at night the number of persons with class D1 decreased comparing to daytime $\left(\chi_{2 \mathrm{MN}}=8.6, \mathrm{p}=0.003\right)$, what leaded to increase of the number of persons with class D2 (Fig. 4). We evaluate this fact as positive, because class D1 is dangerous due to acute hypoten- sive episodes [10,11], class D2 means normotensive hemodynamics although of dysfunctional type (the work of left ventricle is disproportionally increased).

The differences between class H3 frequency in responders and in non-responders allowed assessing the link between this hemodynamic phenotype in daytime period of ABPM and positive response of diurnal index at CPAP therapy: RR (relative risk) $=9.6(95 \%$ CI 1.4-68.3), $0.001<\mathrm{p}<0.01$. It means that the patients with hemodynamic phenotype $\mathrm{H} 3$ in daytime period of baseline ABPM have better chances to a positive response to CPAP treatment $(\mathrm{p}<0.05)$.

There was a significant transformation of models between observation periods of ABPM in non-responders: daytime - nighttime, daytime $-24 \mathrm{~h}$ (Fig. 4). Obviously, it is caused by circulatory changes at night. Five patients (non-responders) changed classes H0, D2 and D3 (on daytime model) into class H3 (on nighttime and 24h models) whereas responders had not such negative transformations. This result was statistically significant: $\chi_{2 \mathrm{MN}}=11.0$, $\mathrm{p}=0.000$.

However, some non-responders also had some positive result from CPAP therapy, which was concluded in transformation of class D1 at daytime period into better hemodynamic classes at night and at $24 \mathrm{~h}$, first into class D2. The difference in diurnal dynamic of this feature between non-responders and responders was significant: $\chi_{2 \mathrm{MN}}=12.1, \mathrm{p}<0.001$.

\section{Conclusion}

Our study showed that individual modelling of hemodynamics by IMH-BP technique may partly predict the BP response in patients with OSA who initiate CPAP therapy.

\section{References}

1. Haentjens P, Van Meerhaeghe A, Moscariello A, et al. The impact of continuous positive airway pressure on blood pressure in patients with obstructive sleep apnea syndrome: evidence from a meta-analysis of placebo-controlled randomized trials. Arch Intern Med. 2007; 167(8): 757-764, doi: 10.1001/ archinte.167.8.757, indexed in Pubmed: 17452537.

2. Baguet JP, Hammer L, Lévy P, et al. Night-time and diastolic hypertension are common and underestimated conditions in newly diagnosed apnoeic patients. J Hypertens. 2005; 23(3): 521-527, indexed in Pubmed: 15716692.

3. Gami AS, Pressman G, Caples SM, et al. Association of atrial fibrillation and obstructive sleep apnea. Circulation. 2004; 110(4): 364-367, doi: 10.1161/01.CIR.0000136587.68725.8E, indexed in Pubmed: 15249509.

4. Martínez-García MA, Galiano-Blancart R, Román-Sánchez P, et al. Continuous positive airway pressure treatment in sleep apnea 
prevents new vascular events after ischemic stroke. Chest. 2005; 128(4): 2123-2129, doi: 10.1378/chest.128.4.2123, indexed in Pubmed: 16236864.

5. Young T, Finn L, Peppard PE, et al. Sleep disordered breathing and mortality: eighteen-year follow-up of the Wisconsin sleep cohort. Sleep. 2008; 31(8): 1071-1078, indexed in Pubmed: 18714778.

6. Gami AS, Olson EJ, Shen WK, et al. Obstructive sleep apnea and the risk of sudden cardiac death: a longitudinal study of 10,701 adults. J Am Coll Cardiol. 2013; 62(7): 610-616, doi: 10.1016/j.jacc.2013.04.080, indexed in Pubmed: 23770166.

7. Peker Y, Hedner J, Kraiczi H, et al. Respiratory disturbance index: an independent predictor of mortality in coronary artery disease.
Am J Respir Crit Care Med. 2000; 162(1): 81-86, doi: 10.1164/ ajrccm.162.1.9905035, indexed in Pubmed: 10903224.

8. Khursa R. Blood pressure: new diagnostic possibilities of the "routine» indicator. Arterial Hypertens. 2014; 18(2): 104-105.

9. Voitikova MV, Khursa RV. Analysis of 24-hour ambulatory blood pressure monitoring data using support vector machine. Nonlinear Phenomena in Complex Systems. 2014; 17(1): 50-56.

10. Khursa RV, Voitikova MV. [Classification of the hemodynamic states on the data of regression modeling of the blood pressure parameters] (in Russian). Clinical Physiology of Blood Circulation. 2013(2): 35-54.

11. Voitikova MV, Khursa RV. Linear regression in hemodynamics. Nonlinear Phenomena in Complex Systems. 2012; 15(2): 203-206. 